\title{
ABUNDANCE SIGNATURES OF INTERNAL STELLAR STRUCTURE IN F STARS
}

\author{
Ann Merchant Boesgaard \\ Palomar Observatory and Department of Astronomy \\ California Institute of Technology
}

\begin{abstract}
We have determined $\mathrm{Li}$ abundances from high resolution, high signalto-noise spectra near $6700 \AA$ of the F dwarfs in several galactic clusters and in the field. The Li-temperature profiles show large $\mathrm{Li}$ depletions in the mid-F stars indicating that matter has been circulated (or diffused) downward in the stars. This brings new observational evidence about the details of stellar envelope structure. $\mathrm{Li}$ depletions occur in stars with $\mathrm{T}=6400-6900 \mathrm{~K}$, with depletions of over an order of magnitude for those with $\mathrm{T}=6500-6800 \mathrm{~K}$. The relationship to rotation, galactic $\mathrm{Li}$, and the halo star $\mathrm{Li}$ content is discussed.
\end{abstract}

\section{INTRODUCTION}

The surface contents of both $\mathrm{Li}$ and $\mathrm{Be}$ provide sensitive probes to the internal structure of stars. For example, the Sun destroys $\mathrm{Li}$ by $(\mathrm{p}, \alpha)$ type reactions at $\sim 2.5 \times 10^{6} \mathrm{~K}$ and has lost 99 percent of its $\mathrm{Li}$, while it burns $\mathrm{Be}$ at $\sim 3.5 \times 10^{6}$ $\mathrm{K}$ and has lost none of its original Be apparently. The temperature at the base of the solar convection zone in solar models is only $1.9 \times 10^{6} \mathrm{~K}$ (D'Antona and Mazzitelli 1984), not enough for $\mathrm{Li}$ (or Be) destruction. For a hotter star, 1.25 $M_{\odot}$, the stellar model is only $6 \times 10^{5} \mathrm{~K}$ at the bottom of the surface convection zone. Thus, according to the models, convection alone does not circulate $\mathrm{Li}$ and $\mathrm{Be}$ to regions of the star where they can be destroyed. However, some main sequence stars of $\geq 1 M_{\odot}$ do show depletion of $\mathrm{Li}$ or of both $\mathrm{Li}$ and $\mathrm{Be}$, so other mixing mechanisms are required.

\section{OBSERVATIONS}

Spectra for a number of $\mathrm{F}$ main-sequence stars in open clusters and in the field have been obtained near the $6700 \AA$ region at the $3.6 \mathrm{~m}$ Canada-France-Hawaii telescope on Mauna Kea and at the $5 \mathrm{~m}$ Hale telescope at Palomar. Those from the CFHT are Reticon spectra from the f/8.2 coudé camera and cover $135 \AA$ with a spectral resolution of $0.11 \AA$ and typical $S / N$ values of $350-600$. Those from Palomar are TI CCD spectra from the 72-inch coudé camera and cover $110 \AA$ with $0.21 \AA$ resolution and $300-500$ in $\mathrm{S} / \mathrm{N}$. Because of the high signal-to-noise ratios, highly accurate $\mathrm{Li}$ abundances can be determined and, for stars with no detectable $\mathrm{Li}$ line, very low upper limits can be ascertained which correspond to very large (2 
- 3 orders of magnitude) $\mathrm{Li}$ depletions.

The spectra have been flat-fielded, wavelength-adjusted, and continuumflattened according to the usual procedures. (See Boesgaard and Tripicco 1986b and Boesgaard, Budge, and Burck 1988) for details.) Equivalent widths or upper limits have been measured for Li I $\lambda 6707$ and for several Fe I lines. A weak Fe I line $(2-4 \mathrm{~m} \AA)$ blends with the $\mathrm{Li}$ I line on the short wavelength side; to account properly for that in the calculations, the abundance of $\mathrm{Fe} / \mathrm{H}$ must be found.

\section{ABUNDANCE RESULTS}

Stellar temperatures have been determined through use of several photometric indices and calibrations. Models of Kurucz (1979) have been used with a model atmosphere abundance routine to predict the $\mathrm{Li} \mathrm{I}$ (and $\mathrm{Li} \mathrm{I}+\mathrm{Fe} \mathrm{I}$ ) equivalent widths for various abundances.

Figure 1 shows the $\mathrm{Li}$-temperature profile for the $\mathrm{F}$ field stars (data from Boesgaard and Tripicco 1986b), the Hyades F dwarfs (primarily from Boesgaard and Tripicco 1986a), the Coma F dwarfs (from Boesgaard 1987a), and for the UMa Group F stars (from Boesgaard, Budge, and Burck 1988). The three open clusters
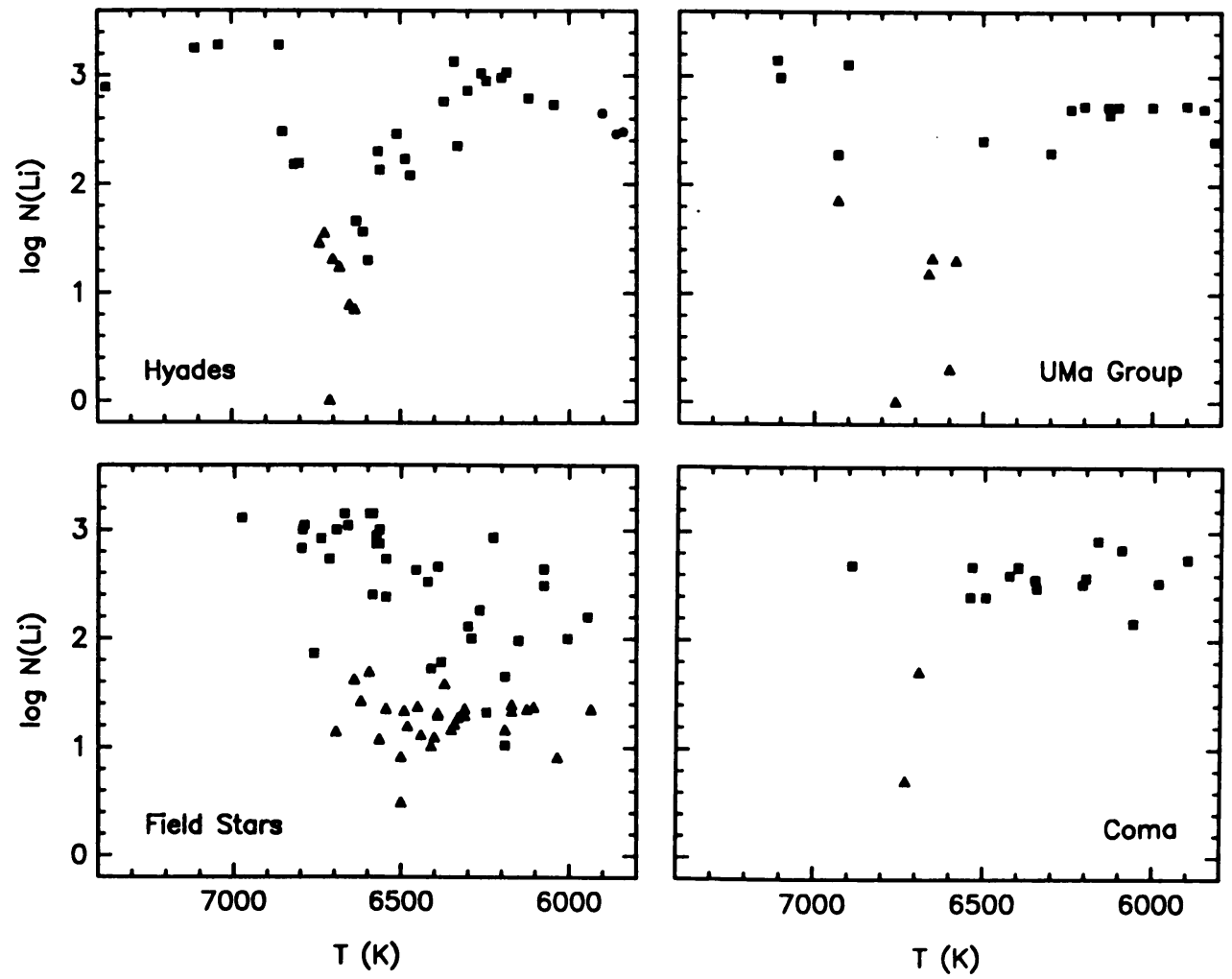

Figure 1. The Li-temperature profile for $\mathrm{F}$ field dwarfs, the Hyades $\mathrm{F}$ dwarfs, the Coma F dwarfs and the UMa F dwarfs. Triangles represent upper limits. 
(ages $7 \times 10^{8}, 5 \times 10^{8}$, and $3 \times 10^{8} \mathrm{yr}$, respectively) all show a pronounced drop in $\mathrm{Li}$ content in the middle $\mathrm{F}$ star range, near temperatures $6400-6900 \mathrm{~K}$, or in stars about 20 percent more massive than the sun. The field stars are not all affected by this phenomenon: about half of the stars in this temperature regime show the normal Pop I abundance, $\mathrm{Li} / \mathrm{H}=10^{-9}$ or $\log \mathrm{N}(\mathrm{Li})=3.0$, and about half show large $\mathrm{Li}$ depletions.

An interpretation of the Hyades $\mathrm{F}$ star $\mathrm{Li}$ depletions in terms of diffusion has been put forward by Michaud (1986). His calculations show that chemical separation takes place below the convection zone because the upward radiative acceleration on $\mathrm{Li}$ is less than the downward acceleration of gravity for the middle$\mathrm{F}$ stars. For the cooler $\mathrm{F}$ stars the diffusion time scale is too long to see the effect in the $7 \times 10^{8} \mathrm{yr}$ lifetime of the Hyades cluster. His calculations do not match the observations in two ways: they predict that $\mathrm{Li}$ will be supported in the early $\mathrm{F}$ stars resulting in overabundances of $\mathrm{Li}$ and they predict only an order of magnitude or so for $\mathrm{Li}$ depletions while more than two orders of magnitude are found. Both of these effects can be reconciled with the observations if the stars are losing mass at rates of about $10^{-14}-10^{-15} M_{\odot} \mathrm{yr}^{-1}$.

It is possible that complex circulation patterns are present in the stellar envelopes relating to rotation, differential rotation and magnetic fields. Figure 2 shows the drop in the measured $v$ sin $i$ values (from Kraft 1965) with temperature over the $\mathrm{Li}$ "chasm" in the Hyades. As one goes toward cooler temperatures in the $\mathrm{F}$ dwarfs the stellar rotation declines and the outer convection zone deepens. The interplay between these two phenomena may result in the sudden drop in the surface $\mathrm{Li}$ content followed by the slower rise. In the $\mathrm{Li}$ chasm it appears that the rapid rotators are more severely depleted in $\mathrm{Li}$.
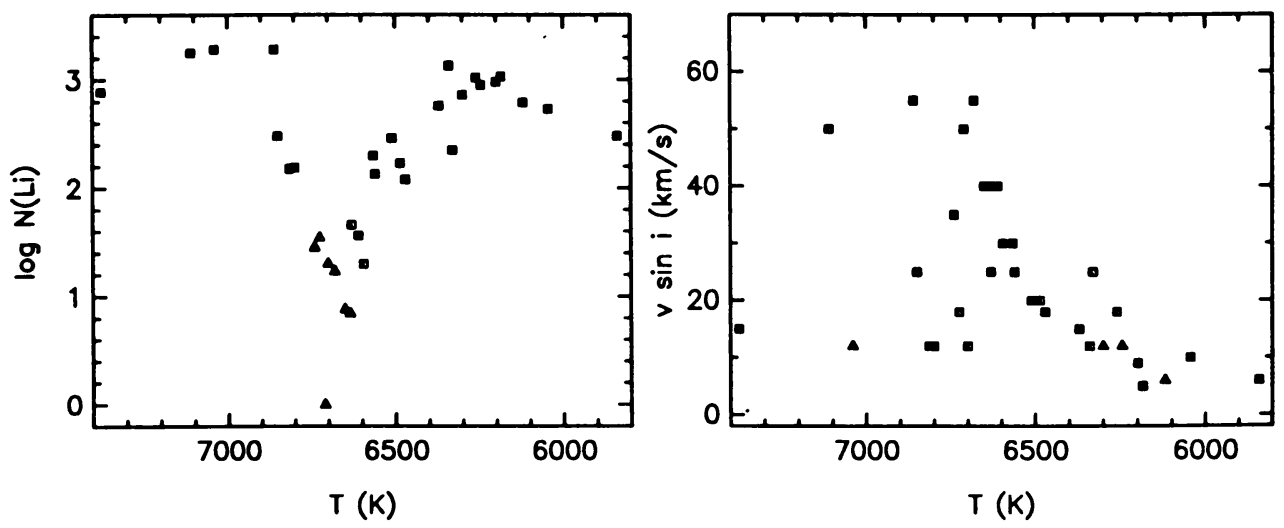

Figure 2. The Li-temperature and $v$ sin $i$-temperature profiles for the Hyades $\mathrm{F}$ dwarfs.

\section{DISCUSSION AND CONCLUSIONS}

All the galactic clusters appear to have the same amount of $\mathrm{Li}$ as shown by the $\mathrm{F}$ dwarfs that are hotter than the mid-F star $\mathrm{Li}$ gap, $\log \mathrm{N}(\mathrm{Li}) \sim 3.0$. This is the same amount as seen in the chondrites (Nichiporuk and Moore 1974), the F field stars (Boesgaard and Tripicco 1986b), F and $\mathrm{G}$ visual binaries (Boesgaard 
and Tripicco 1987) and T Tauri stars (Mundt et al. 1983). These objects span the age range from $\sim 10^{6}$ to $5 \times 10^{9} \mathrm{yr}$. From this we can infer that there has been no measurable enrichment of $\mathrm{Li}$ in the galactic disk since the formation of the solar system.

The four galactic clusters in which $\mathrm{Li}$ in the $\mathrm{F}$ dwarfs has been recently studied - UMa at $3 \times 10^{8}$ yr (Boesgaard, Budge, and Burck 1988), Coma at $5 \mathrm{x}$ $10^{9} \mathrm{yr}$ (Boesgaard 1987a), Hyades at $7 \times 10^{8} \mathrm{yr}$ (Boesgaard and Tripicco 1986a and Boesgaard 1987b) and NGC 752 at $2 \times 10^{9}$ yr (Hobbs and Pilachowski 1986) - all show evidence of the deep $\mathrm{Li}$ depletions in the mid-F stars first shown by Boesgaard and Tripicco (1986a) in the Hyades. In addition, visual binaries with ages of $8 \times 10^{8}-3 \times 10^{9}$ yr also show this effect. The "Li chasm" occurs at B $\mathrm{V}=0.40-0.47$, or $\mathrm{T} \approx 6400-6900 \mathrm{~K}$. The chasm has a width of only $300 \mathrm{~K}$ at the $\log \mathrm{N}(\mathrm{Li})=2.0$ level.

In the Hyades the bottom of the $\mathrm{Li}$ gap is at $\mathrm{T} \sim 6650 \mathrm{~K}$ where the depletions of $\mathrm{Li}$ are at least 3 orders of magnitude. As the the temperature decreases, the value of $\log \mathrm{N}(\mathrm{Li})$ increases and $v \sin i$ decreases. For the stars in the Hyades $\mathrm{Li}$ chasm there appaers to be an inverse relation between $\log \mathrm{N}(\mathrm{Li})$ and $v$ sin $i$ such that the more rapidly rotating stars are more depleted in $\mathrm{Li}$.

On the cool side of the $\mathrm{Li}$ dip the three clusters that have ages near $5 \mathrm{x}$ $10^{8}$ show a flat distribution of $\mathrm{Li}$ vs. temperature with a $\log \mathrm{N}(\mathrm{Li})$ value of $\sim 2.7$, slightly below the initial value found in the F stars on the hot side of the $\mathrm{Li}$ chasm. These stars have apparently undergone some $\mathrm{Li}$ depletion, the amount of which may be age related but is not very mass dependent. This flatness from $T \sim 5800$ $-6300 \mathrm{~K}$ is reminiscent of the flatness in the $\mathrm{Li}$-temperature plot for the halo dwarfs. (See Figure 3.) The similarities in the flatness and in the spread of log $\mathrm{N}(\mathrm{Li})$ values at a given temperature indicate that it is possible that the halo stars have undergone $\mathrm{Li}$ depletion (diffusion?) during their long lifetimes from an initial level higher than that presently observed.

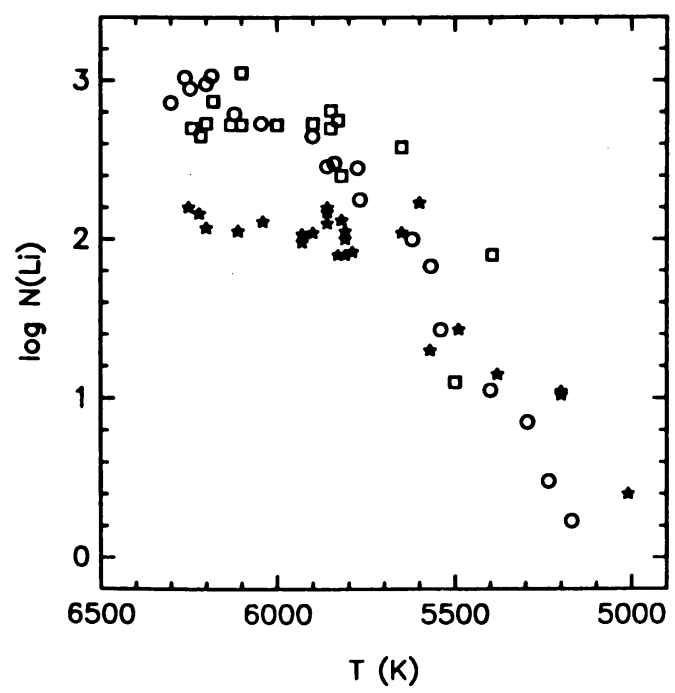

Figure 3. The Li-temperature profile for the late $\mathrm{F}$ and $\mathrm{G}$ dwarfs of the Hyades (open circles), the UMa group (open squares) and the halo dwarfs(filled stars). 
The data for the halo stars in Figure 3 are only for those with velocities (relative to the local standard of rest) $\geq \pm 100 \mathrm{~km} \mathrm{~s}^{-1}$ and with $[\mathrm{Fe} / \mathrm{H}] \leq-1.4$; the temperature and $\mathrm{Li}$ values are from Spite, Maillard, and Spite (1984), Spite and Spite (1986), and Hobbs and Duncan (1987). The Hyades values are from Boesgaard (1987b) and those for UMa from Boesgaard et al. . (1988).

The complex Li-temperature profile found for F and $\mathrm{G}$ dwarfs in galactic clusters indicates that the internal stellar structure and processes are more complicated than had been recognized. Calculations of the internal circulation of matter (certainly of $\mathrm{Li}$ atoms) caused by stellar rotation, especially differential rotation, are needed as are more sophisticated calculations of the effects of diffusion in both young stars and in halo stars.

I am happy to acknowledge support from NSF grants, AST-8216192 and RII-8521715, and a Guggenheim Fellowship and the concomitant hospitality of the California Institute of Technology.

\section{REFERENCES}

Boesgaard, A. M. 1987a, Astrophys. J., 321, in press.

Boesgaard, A. M. 1987b, Publ. A. S. P., 99, in press.

Boesgaard, A. M., and Trippico, M. J. 1986a, Astrophys. J., 302, L49.

Boesgaard, A. M., and Trippico, M. J. 1986b, Astrophys. J., 303, 724.

Boesgaard, A. M., and Trippico, M. J. 1987, Astrophys. J., 313, 389.

Boesgaard, A. M., Budge, K. G., and Burck, E. E. 1988, Astrophys. J., 325, in press.

D'Antona, F. and Mazzitelli, I. 1984, Astr. Ap., 138, 431.

Hobbs, L. M. and Pilachowski, C. A. 1986, Astrophys. J., 309, L17.

Hobbs, L. M. and Duncan, D. K. 1987, Astrophys. J., 317, 796.

Kraft, R. P. 1965, Astrophys. J., 142, 681.

Kurucz, R. L. 1979, Astrophys. J. Suppl., 40, 1.

Michaud, G. 1986, Astrophys. J., 302, 650.

Mundt, R., Walter, F. M., Feigelson, E. D., Finkenzeller, U., Herbig, G. H., and Odell, A. P. 1984, Astrophys. J., 269, 229.

Nichiporuk, W. and Moore, C. B. 1974, Geoch. et Cosmoch. Acta, 38, 1691.

Spite, M. Maillard, J.-P., and Spite, F. 1984, Astr. Ap., 141, 56.

Spite, F. and Spite, M. 1986, Astr. Ap., 163, 140. 


\section{Drscussion}

SNEDEN Could you please expand on your points suggesting a) a constancy in Li over the age of the galactic disk, and b) the apparent depletion of $\mathrm{Li}$ in halo stars.

BOESGAARD The "initial" Li abundance, which all the stars in a cluster are born with, must be determined from the $F$ dwarfs on the hot side of the Li dip in the mid-P dwarfs. These values $-\log N(\mathrm{Li})=3.0$ - are the same from cluster to cluster and the same as the $T$ Tau stars and the solar system values. On the cool side of the Li dip, T=5800-6400 K, there seems to be only very weak dependance of $\mathrm{Li}$ on temperature, the stars in the three clusters, Hyades, Coma, and uxa (with ages 5x100 yrs.) show depleted Li $-\log N(L i)=2.7$. The older cluster, NGC 752 (with age $~ 2 x 100$ yrs.) shows $-\log N(\mathrm{Li})=2.4$ in this temperature regime. The halo stars at $\log N(L i)=2.1$ and age $~ 12 \times 10^{\circ}$ Yrs. may be showing a similar depletion with age in this temperature region.

Ann Boesgaard.

P. SPITE First my congratulations for the beautiful work of

About the comparison of the flat paxt of clusters and of pop. II dwarfs : if it is expected to find a plateau with small scatter for a cluster, in which all stars have the same age and metallicity, it is more difficult to understand for pop.II dwarfs which may have the same age but widely different metallicities. And up to now, the theories of lithium depletion predict depletion rates dependent on metallicities.

BOESGAARD However, there does seem to be a real spread in the Li abundances at a given temperature in the halo stars. This indicates that some depletion may have occured. All the halo stars plotted have $[\mathrm{Pe} / \mathrm{H}]<-1.4$.

DUNCAN Comment on F. Spite's question.

I suggest waiting until this afternoon, when I will discuss if star to star variations are real, and the effect of $[\mathrm{Pe} / \mathrm{H}]$ differences on $\mathrm{Li}$ destruction rate.

NISSEN There has also ben observation of $\mathrm{Li}$ in $\mathrm{F}$ stars in the old, open cluster 467 (Pilachowski et al., and Spite et al.). Do these results fit your $\mathrm{Li}$ - age relation for the plateau around $\mathrm{T}_{\text {eff }} \approx 6000$ $6400 \times ?$

BOESGAARD The hottest main-sequence stars (late $F$ dwarfs) left in 467 barely reach the plateau region. However, these authors have observed six stars in this temperature range with $\log N(\mathrm{Li})$ values all near 2.5 . 